\title{
Diensthabende
}

\section{Von Schubladen und Menschen}

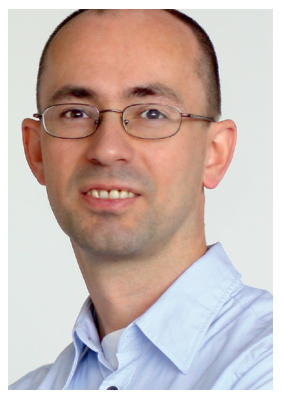

Schon mal was vom Pallister-Killian-Syndrom gehört? Von der Medionecrosis cystica aortae? Oder von der Mukopolysaccharidose PfaundlerHurler? Die Ärzte, denen wir unser aktuelles Titelthema (S.24) widmen, kennen sie alle! Und zwar nicht nur dem Namen nach - sondern gewissermaßen „persönlich“. Pathologen sind Experten darin, jeder auch noch so abgefahrenen Krankheit mittels Immunhistologie, Gensequenzierung \& Co. auf die Schliche zu kommen - und sie zu klassifizieren. Dieses Einsortieren in „Schubladen“ ist die Grundlage der modernen Medizin. Denn nur so kann eine Krankheit gezielt bekämpft werden. Doch so toll und wichtig der Job ist, den die Pathologen da tun. Manchmal beschleicht mich bei all der Klassifiziererei auch ein ungutes Gefühl. Berücksichtigt jeder Arzt, dass eine Diagnose nie die ganze Wahrheit über einen Menschen erzählt? Und dass zwei gleiche Diagnosen nicht bedeuten, dass die Patienten auch dieselben Bedürfnisse haben? Hoffnung gibt mir da unsere kleine Umfrage zum Thema „Träum dir was!“(S. 18). Gefragt, wie sie als Arzt später sein möchten, betonten die meisten Jungmediziner, es sei ihnen wichtig, dass sie sich für die Sorgen ihrer Patienten Zeit nehmen können. Ist das repräsentativ für die Mehrheit der angehenden Ärzte, ist mir für die Zukunft unserer Medizin nicht bange.

Herzlichst

euer Dieter Schmid

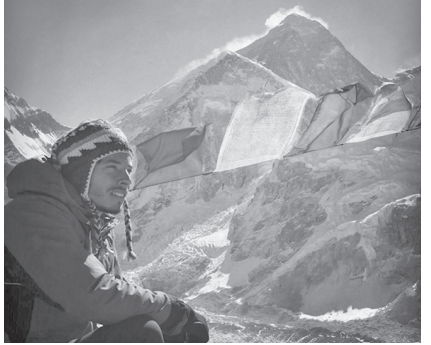

Simon Witthauer

zog es für eine Famulatur in die abgelegensten Gebiete Nepals, einem Land, das sich der modernen westlichen Medizin nur sehr langsam annähert. In seinem Bericht erzählt der Medizinstudent und Physiotherapeut von seinen - nicht nur geografisch bedingt - extremen Erfahrungen (S. 14).

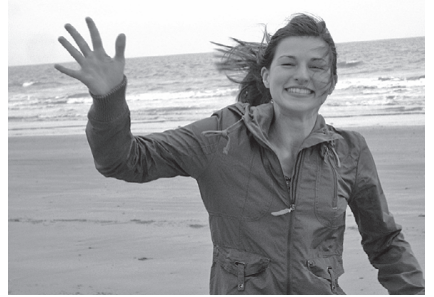

Sarah Böhmer

findet, dass man als junger Assistenzarzt manchmal ganz schön ins kalte Wasser geschmissen wird - vor allem bei brenzligen Situationen in Nachtdiensten. In diesem Heft gibt sie euch einen Fahrplan für die drei häufigsten Leitsymptome, die in Nachtdiensten „aufschlagen“ (S. 46).

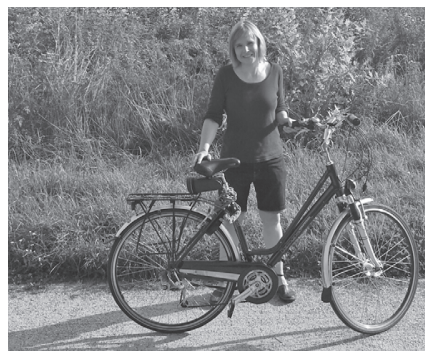

Silja Schwencke

sieht in der Geriatrie bei bakteriellen Infekten häufig schwere Verläufe, bei denen auch Reserve-Antibiotika nicht mehr helfen. Trotzdem hat die Allgemeinärztin beim Schreiben ihres Artikels über Antibiotika fast Sympathie entwickelt für die Überlebenskraft dieser kleinen „infektiösen Schufte“ (S. 34). 